\title{
BMJ Open Single and mixed dyslipidaemia in Canadian primary care settings: findings from the Canadian primary care sentinel surveillance network database
}

\author{
Shabnam Asghari, ${ }^{1}$ Erfan Aref-Eshghi, ${ }^{1}$ Marshall Godwin, ${ }^{1}$ Pauline Duke, ${ }^{1}$ \\ Tyler Williamson, ${ }^{2}$ Masoud Mahdavian ${ }^{3}$
}

To cite: Asghari S, ArefEshghi E, Godwin M, et al. Single and mixed dyslipidaemia in Canadian primary care settings: findings from the Canadian primary care sentinel surveillance network database. BMJ Open 2015;5: e007954. doi:10.1136/ bmjopen-2015-007954

- Prepublication history for this paper is available online. To view these files please visit the journal online (http://dx.doi.org/10.1136/ bmjopen-2015-007954).

Received 15 February 2015 Revised 30 September 2015 Accepted 16 October 2015

CrossMark

\footnotetext{
${ }^{1}$ Faculty of Medicine, Memorial University of Newfoundland, St. John's, Newfoundland and Labrador, Canada

${ }^{2}$ Department of Community Health Sciences, University of Calgary, Calgary, Alberta, Canada

${ }^{3}$ Faculty of Medicine, University of Ottawa, Ottawa, Ontario, Canada
}

Correspondence to Dr Shabnam Asghari; shabnam.asghari@med. mun.ca

\section{ABSTRACT}

Objectives: Dyslipidaemia is a major risk factor to cardiovascular disease (CVD) - the leading cause of death worldwide. Limited data are available about the prevalence of various dyslipidaemia in Canada. The objective of this study is to describe the prevalence of various single and mixed dyslipidaemia within the Canadian population in a primary care setting.

Setting: A cross-sectional study, using the Canadian Primary Care Sentinel Surveillance Network (CPCSSN), was undertaken.

Participants: Non-pregnant adults older than 20 years were included.

Outcome measures: Canadian guidelines were used to define dyslipidaemia. Descriptive statistics and multivariate regression analyses were conducted to compare the prevalence of single/mixed dyslipidaemia.

Results: 134074 individuals with a mean age of 59.2 (55.8\% women) were identified. $34.8 \%$ of this population had no lipid abnormality, whereas $35.8 \%$, $17.3 \%$ and $3.2 \%$ had abnormalities in one, two and three lipid components, respectively. Approximately $86 \%$ of these patients did not receive any lipidlowering medication. Among the medication users (14\%), approximately $12 \%$ were on statin monotherapy. Statin users $(n=16036)$ had a lower rate of low-density lipoprotein dyslipidaemia compared to non-medication users ( $3 \%$ vs $17 \%)$, whereas the prevalence of high-density lipoprotein (HDL) $(20 \%$ vs $12 \%$ ) and triglycerides (TG) (12\% vs $7 \%$ ) dyslipidaemia were higher in statin users. Statin users had a greater prevalence of HDL, TG and combined HDL-TG dyslipidaemia compared to non-medication users (OR 1.44, 95\% $\mathrm{Cl} 1.36$ to 153), (OR 1.18, 95\% $\mathrm{Cl} 1.10$ to 1.27 ) and (OR $1.30,95 \% \mathrm{Cl} 1.22$ to 1.38$)$, respectively, (all $p$ values $<0.0001$ ).

Conclusions: One of every five patients in primary care settings in Canada is suffering from mixed dyslipidaemia. The overall prevalence of dyslipidaemia remains the same between treated and untreated groups, although the type of abnormal lipid component is considerably different. Among the CVD risk factors,

\section{Strengths and limitations of this study}

- To our knowledge, this is the first report describing single and mixed dyslipidaemia based on biochemical measurements of lipids in a large Canadian population.

- This study includes a large population of patients who visited family physicians across Canada within a 3-year period.

- The study describes the prevalence of various lipid disorders among individuals treated in accordance with Canadian guidelines. One of every five patients in primary care settings in Canada is suffering from mixed dyslipidaemia.

- The cross-sectional nature of the study does not provide insight on the patients' adherence to medication therapy.

- The study does not have any data on some of the life-style factors that are associated with lipid levels, such as diet and physical activity.

obesity has the greatest effect on the prevalence of all types of dyslipidaemia.

\section{INTRODUCTION}

In 2012, ischaemic heart disease and stroke (cardiovascular diseases-CVDs), were ranked together as the first cause of mortality with more than 14 million deaths worldwide. ${ }^{1}$ The impact of CVDs in this mortality rate is more significant in countries with higher incomes. ${ }^{1}$ In Canada, CVDs are responsible for $32 \%$ of deaths and its economic burden is estimated to be second only to musculoskeletal conditions. ${ }^{2}$ Epidemiological studies have identified age, male gender, cigarette smoking, diabetes mellitus, dyslipidaemia, hypertension, obesity and a family history of CVDs as major risk factors contributing to 
the majority of CVDs. ${ }^{3}$ Among these risk factors, dyslipidaemia, defined as abnormal blood lipid levels that include elevated total cholesterol (TC), low-density lipoprotein (LDL-C), triglycerides (TG) and decreased highdensity lipoprotein (HDL), is considered as one of the most important and the easiest modifiable CVDs. ${ }^{5}$

LDL-C has long been presented as the major lipid component involved in the risk of CVDs. ${ }^{6}$ However, low serum levels of HDL-C, as well as high levels of TG, can also contribute to the risk of CVDs, irrespective of LDL-C levels; ${ }^{7-9}$ these two lipid components tend to be in correlation with each other. ${ }^{10}$ It is important, therefore, to consider the complete picture (which is composed of all serum lipids) when treating individuals who are at high risk.

Current CVDs prevention guidelines primarily focus on lowering LDL-C as the main goal in the treatment of dyslipidaemia. $^{211}$ According to these guidelines, statin therapy, which is recommended as the treatment of choice for the management of elevated LDL-C, is not sufficient for patients suffering from a mixed dyslipidaemia-a combined elevation in LDL and TG levels that may be accompanied by low levels of HDL cholesterol $^{12}$ - those patients remain at substantial risk for developing CVDs. ${ }^{13}$ Prospective trials in acute coronary syndrome and stable patients with chronic heart disease have shown that elevated plasma levels of TG and low plasma concentrations of HDL-C are associated with high risks of recurrent CVD events, even at or below recommended LDL-C goals. ${ }^{14-16}$ As well, HDL-C has been identified as the second most important coronary risk factor after LDL-C in patients with type II diabetes. ${ }^{17}$ Therefore, to further address this residual risk, alternative guidelines have suggested that HDL-C and TG be managed among high-risk individuals and that other medications, such as niacin and fibrates, or a combination medication therapy be used. ${ }^{18}$

The prevalence of dyslipidaemia is reported to be high in Canada and the status of primary care management for lipid disorders does not appear to be sufficient. The Canadian Health Measure Survey (CHMS) reports that $45 \%$ of Canadian adults have dyslipidaemia among whom $57 \%$ are not aware of their condition. ${ }^{19}$ In a large cohort study representing Canadian primary care practice, only $36 \%$ of those with abnormal levels of lipid were receiving treatment for dyslipidaemia. ${ }^{20}$ Despite reports, very little is known about the status of mixed dyslipidaemia in Canada. In describing the prevalence of dyslipidaemia in general and those with certain conditions imposing a higher risk of CVDs, including those with diabetes, a history of CVDs and the multiple risk factors will assist in CVD prevention. This information will also improve the quality of care for these groups through consideration of targeted therapies of mixed dyslipidaemia by either pharmacological or nonpharmacological measures. ${ }^{21}$ This is of particular importance, given that the mechanism underlying mixed dyslipidaemia is different from single dyslipidaemia and mainly consists of hepatic overproduction of very low density lipoprotein particles, which lead to increases in TG and LDL cholesterol. ${ }^{22}$ The objective of this study, therefore, is to describe the prevalence of different types of lipid disorders among the general primary care Canadian population and those under treatment in accordance with Canadian guidelines. The association between CVD risk factors, including patients' characteristics and comorbidities, and various types of dyslipidaemia was also examined.

\section{METHODS}

\section{Source of data}

A cross-sectional study, using data from the Canadian Primary Care Sentinel Surveillance Network (CPCSSN) database, was undertaken. ${ }^{23}$ This database contains electronic medical records of family physicians (EMRs) which are abstracted quarterly and uploaded to a de-identified system to regional and central pan-Canadian databases. The CPCSSN database is used for examining chronic diseases in primary care as well as for primary care research. ${ }^{24}$ At the time of this study, the pan-Canadian CPCSSN database included data from approximately 600 primary-care clinicians in rural and urban settings across 10 Canadian provinces, and involved medical records of 844592 individuals over 20 years of age (equal to $3 \%$ of the Canadian population over the age of 20 years).

\section{Study population}

The study population includes non-pregnant adults older than 20 years of age who had a lipid profile in the CPCSSN database between 1 January 2010 and 31 December 2012. For identification and removal of pregnant women from the cohort, the text and/or ICD code records for every event related to pregnancy were queried. ${ }^{26}$

\section{Variables}

\section{Lipid variables}

Canadian guidelines for the diagnosis and management of dyslipidaemia, the nationwide protocol for all practitioners in Canada, suggest a lipid screen for various age groups; that is, all men over the age of 40, all women over the age of 50 , first-degree relatives with a history of CVD under the age of 60, all postmenopausal women, all individuals with diabetes, hypertension, obesity and current smokers. The routine screening test requires the measurement of all lipid components. In this study, the most recent lipid profiles (TC, HDL, LDL, and TG) for each individual were recorded. The ratio of TC to HDL was calculated by dividing the TC by HDL. Dyslipidaemia was defined using Canadian Guidelines for the Diagnosis and Treatment of Dyslipidemia (table 1). ${ }^{11}$ Mutually exclusive dyslipidaemia (single dyslipidaemia) was defined as the existence of only one abnormal lipid element in the individual; whereas 
Table 1 Healthy levels of serum lipids for Canadian adults (20-79 years of age $)^{11}$

\begin{tabular}{ll}
\hline Lipid component & Normal levels \\
\hline Total cholesterol (TC) & $<5.2 \mathrm{mmol} / \mathrm{L}$ \\
Triglycerides (TG) & $<1.7 \mathrm{mmol} / \mathrm{L}$ \\
Low-density lipoprotein (LDL) & $<3.4 \mathrm{mmol} / \mathrm{L}$ \\
cholesterol & $>1.0 \mathrm{mmol} / \mathrm{L}$ men; \\
High-density lipoprotein (HDL) & $>1.3 \mathrm{mmol} / \mathrm{L}$ women \\
cholesterol & $<5.0$ \\
Ratio of TC to HDL &
\end{tabular}

mixed dyslipidaemia was defined as the existence of more than one lipid disorder. In the initial descriptive statistics of the study population, all five components were presented; however, in the subsequent analyses, the TC and ratio were not considered as they both contained elements of the other three components.

\section{Demographic variables}

The demographic variables including age, gender and place of residence were extracted from the database. The rural/urban residence was determined by using the second character of each individual's postal code address. ${ }^{27}$ The rural/urban residence was included in the multivariate analysis as previous studies suggest that the CVD risk factors could differ between the rural and urban inhabitants. $^{28}$

\section{Risk factors/comorbid conditions}

The smoking status was extracted from the most recent record by the family physician at the time of the lipid test, and individuals were classified as non-smokers, past smokers and current smokers according to the text report and ICD code records in EMRs. Obesity was defined as individuals having a body mass index (BMI) $\geq 30$, whereas those individuals with a BMI lower than 30 , but higher than 25, were classified as being overweight. To ascertain diabetes and hypertension, CPCSSN algorithms for chronic conditions were used. ${ }^{28}$ These definitions have high sensitivity and specificity to detect diabetes and hypertension. ${ }^{29}$ Diagnostic text and ICD code records in EMRs were also used for other chronic conditions, including dyslipidaemia and CVDs. ${ }^{30} 31$

\section{Medication use}

Medication use was identified using the text record and/or Anatomical Therapeutic Chemical (ATC) codes. $^{32}$ The use of lipid modifying agents (HMG-CoA reductase inhibitors, Fibrates, Bile Acid Sequestrants, Nicotinic Acid and other agents) was stratified into two categories: Medication Users (those with any record of lipid lowering agents (LLA) in the database during the 3 months before the date of a blood test); and Non-medication users (those with no record of lipid lowering medication use within 3 months before the date of a blood test). ${ }^{32}$ Among the medication users, those who received statin monotherapy in accordance with Canadian guidelines were separated as a single group, and the status of single and mixed dyslipidaemia among them were compared to those with non-medication therapy. Since only less than $2 \%$ of the medication users in our study were under treatment with lipid modifying agents other than statins, the analyses including lipid-lowering medications focused on statin monotherapy versus non-medication use.

Recent use of other medications with unintended effects on lipid levels, ${ }^{33}$ including thiazides, loop diuretics, $\beta$ blockers, $\alpha$ blockers, ACE inhibitors, calcium channel blockers, oestrogen, progesterone, hormone replacement therapy, and corticosteroids were also extracted from EMRs.

\section{Statistical analyses}

General characteristics of the study population, as well as the prevalence of single and mixed dyslipidaemia among the general population and lipid lowering agent users, were summarised using descriptive statistics and were compared using classical tests of hypothesis including student's $t$ test and the $\chi^{2}$ test. Multivariate multinomial logistic regression modelling was performed to assess dyslipidaemia among lipid-lowering medication users and non-medication users for age, gender, place of residence and other potential influential factors. For variables with more than $5 \%$ of missing information; that is, smoking ( $70 \%$ missing) and BMI $(\sim 50 \%$ missing), a code for missing values was considered wherever model-based analyses were performed; all other variables had missing rates below 5\%. The statistical report was prepared according to the non-missing component of the data. In the multivariate analysis, individuals in the smoking category were compared with non-smokers; overweight and obese patients were compared with normal and underweight individuals; nonmedication users and previous medication users were compared with current medication users as a baseline. A p value of less than 0.05 was considered statistically significant. STATA/IC V.11.2 (Stata Corp., College Station, Texas, USA) was used to perform all the statistical analyses.

\section{Ethics}

The study protocol was approved for ethics by the Health Research Ethics Authority (HREA) of Newfoundland and Labrador. Patient records and information were anonymous and de-identified prior to any analysis.

\section{RESULTS}

\section{Population description}

From 1 January 2010 to 31 December 2012, a total of 430169 individuals were recorded in the CPCSSN database, among which 134074 individuals ( 30\%) had completed a blood test for at least one lipid component 
and met the study criteria. This population had a mean age of $59.2 \pm 15 ; 55 \%$ of them were females, and $23 \%$ were living in rural areas (table 2). A total of $13.82 \%$ of the population $(n=18534)$ were categorised as medication users, among whom the great majority $(n=16036)$ were classified as single statin users (table 2).

The average lipid levels in the study population are presented in table 2. Although the mean lipid levels are at normal ranges, the prevalence of dyslipidaemia for each component (regardless of the existence of other forms of dyslipidaemia) is $40 \%$ for TC, $29 \%$ for HDL, $26 \%$ for LDL, $26 \%$ for TG and $15 \%$ for ratio $>5$.

\section{Single and mixed dyslipidaemia in patients of EMR primary care in Canada}

A total of 111726 individuals had lipid levels available for all three components of HDL, LDL and TG in their EMRs concomitantly; and, hence, were considered for further exploration. In approximately $35 \%$ of this population, all three components were in normal ranges, whereas $36 \%, 17 \%$ and $3 \%$ of the participants had abnormalities in one, two, and three lipid components, respectively. Figure 1 illustrates the prevalence of single (mutually exclusive dyslipidaemia) and mixed

Table 2 Demographic and clinical characteristics of the study population $(n=134074)$

\begin{tabular}{|c|c|}
\hline & $\begin{array}{l}\text { All study } \\
\text { population }\end{array}$ \\
\hline Age $^{*}$ & $59.18 \pm 15.00$ \\
\hline $\mathrm{BMI}^{*}$ & $28.08 \pm 6.40$ \\
\hline Gender $(F)$ & $55.8 \%$ \\
\hline Residence (rural) & $22.7 \%$ \\
\hline Smoking (current)† & $14.2 \%$ \\
\hline Smoking (previous)† & $40.7 \%$ \\
\hline Hypertension & $33.3 \%$ \\
\hline Diabetes mellitus & $15.1 \%$ \\
\hline History of dyslipidaemia & $21.7 \%$ \\
\hline Cardiovascular diseases & $35.4 \%$ \\
\hline Drugs with unintended lipid effects & $22.3 \%$ \\
\hline \multicolumn{2}{|l|}{ Lipid lowering agent use } \\
\hline Non-medication users & $86.2 \%$ \\
\hline Single statin users & $11.9 \%$ \\
\hline Combined medication users & $1.3 \%$ \\
\hline $\begin{array}{l}\text { Single usage of other lipid modifying } \\
\text { agents }\end{array}$ & $0.6 \%$ \\
\hline \multicolumn{2}{|l|}{ Lipid profile } \\
\hline Total cholesterol* & $4.94 \pm 1.09$ \\
\hline $\mathrm{HDL}^{*}$ & $1.39 \pm 0.42$ \\
\hline $\mathrm{LDL}^{*}$ & $2.91 \pm 0.93$ \\
\hline Triglyceride* & $1.42 \pm 0.92$ \\
\hline Total cholesterol/HDL ratio* & $3.79 \pm 1.60$ \\
\hline \multicolumn{2}{|c|}{$\begin{array}{l}\text { Figures are a percentage except for: } \\
{ }^{*} \text { Mean } \pm \text { SD. } \\
\text { †The statistics provided are according to the available information. } \\
\text { Besides smoking and BMI which have missing rates close to } 70 \% \\
\text { and } 50 \% \text {, respectively, the missing rates in all other variables are } \\
\text { below } 5 \% \text { of the total population }(n=134074) \text {. } \\
\text { BMI, body mass index; HDL, high-density lipoprotein; TG, } \\
\text { triglycerides. }\end{array}$} \\
\hline
\end{tabular}

dyslipidaemia among individuals suffering from abnormal levels of LDL, HDL or TG. As can be seen, the most commonly shared abnormality is between those with HDL and TG dyslipidaemia, representing 13\% of the population. Consequently, these patients suffer from a higher rate of mixed dyslipidaemia than those with abnormal levels of LDL.

\section{Comparison of single and mixed dyslipidaemia between} lipid-lowering medication users and non-medication users

The prevalence of overall dyslipidaemia was similar among statin users and non-users (21\% vs $20 \%)$; yet, the pattern was different. As shown in figure 2, approximately $45 \%$ of both groups had all three lipid components within the normal range. Among patients with single dyslipidaemia, the prevalence of high-LDL is lower among statin users ( $3 \%$ vs $17 \%$, $p$ value for $\left.\chi^{2}<0.0001\right)$, whereas the prevalence of HDL and TG dyslipidaemia is significantly $8 \%$ and $5 \%$ higher than untreated participants ( $p$ value from $\chi^{2}<0.0001$ ). Among patients with mixed dyslipidaemia, the combined abnormality of HDL and TG in statin users is almost twice as high as non-users ( $p$ value from $\chi^{2}<0.0001$ ); all other forms of mixed dyslipidaemia that contain LDL are much less prevalent among statin users than non-users.

The differences of TC and ratio dyslipidaemia before exclusion have been calculated in the overall population, both of which had a higher prevalence in nonmedication users compared to statin users (TC $(43.89 \%$ vs $13.57 \%, \mathrm{p}<0.0001)$, and ratio $(15.57 \%$ vs $7.33 \%$, $\mathrm{p}<0.0001))$.

\section{Factors associated with single and mixed dyslipidaemia}

The prevalence of each kind of dyslipidaemia has been stratified by the risk factors associated with CVDs in table 3. table 4 represents the results of the multinomial logistic regression modelling for factors associated with single and mixed dyslipidaemia. As shown in table 4, obese individuals are more likely to have any combination of dyslipidaemia, with the highest OR of 9.39 (95\% CI 8.24 to 10.71) for combined HDL-TG dyslipidaemia, followed by an OR of 9.16 (95\% CI 7.46 to 11.26) for mixed LDL-TG dyslipidaemia. Women are more likely to have dyslipidaemia containing HDL; that is, HDL dyslipidaemia (OR 1.45, 95\% CI 1.39 to 1.51), HDL-LDL dyslipidaemia (OR 2.17, 95\% CI 2.00 to 2.36), and HDL-LDL-TG dyslipidaemia (OR 1.61, 95\% CI 1.50 to 1.74 ), whereas any dyslipidaemia containing TG is more common among men: TG dyslipidaemia (OR 0.55, $95 \%$ CI 0.53 to 0.58 ), and LDL-TG dyslipidaemia OR $0.58,95 \%$ CI 0.55 to 0.62$)$.

After controlling other risk factors, statin users were less likely to have single LDL dyslipidaemia (OR 0.18, $95 \%$ CI 0.16 to 0.20$)$ ) as well as mixed dyslipidaemia of all lipid combinations that contain LDL, compared to non-medication users (LDL-HDL (OR 0.30, 95\% CI 0.24 to 0.36$)$ ); LDL-TG (OR $0.28,95 \%$ CI 0.24 to 0.32 ); LDL-HDL-TG (OR $0.31,95 \%$ CI 0.26 to 0.37 )). 
Figure 1 Prevalence of single and mixed dyslipidaemia of LDL, HDL, and triglycerides in patients of EMR primary care settings in Canada $(n=111726)$. The figure does not account for the possibility of having the dyslipidaemia of total cholesterol or ratio. The sizes of the circles are schematic and do not represent their true values. EMR, electronic medical records of family physicians; HDL, high-density lipoprotein; LDL, low-density lipoprotein.

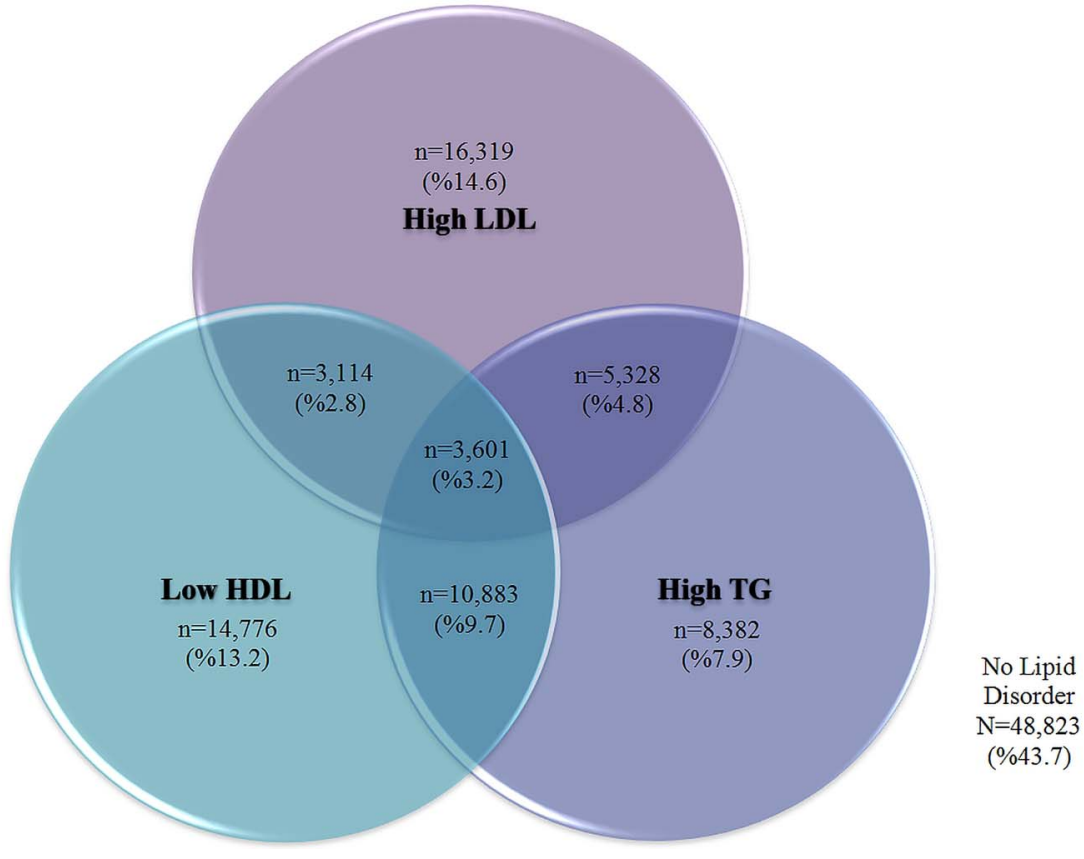

Statin users had a higher rate of single dyslipidaemia of TG (OR 1.18, 95\% CI 1.10 to 1.27 ) and HDL (OR $1.44,95 \%$ CI 1.36 to 1.53$)$ as well as combined HDL-TG dyslipidaemia (OR 1.30, 95\% CI 1.22 to 1.38) compared with non-medication users. No significant effect was observed from medications with unintended lipid effects.

\section{DISCUSSION}

Our study includes a large population of patients who visited family physicians across Canada. To our knowledge, this is the first study describing single and mixed dyslipidaemia based on biochemical measurements of lipids in such a large Canadian population. The results suggest the importance of mixed dyslipidaemia in addition to the single lipid abnormalities in the Canadian population. While a substantial portion of Canadians suffer from various forms of dyslipidaemia, 20.5\% have dyslipidaemia of more than one lipid component simultaneously; this group has a high risk for developing CVDs. $^{7-9} 34$

Mixed dyslipidaemia is an important subject which is not primarily addressed in current guidelines for the

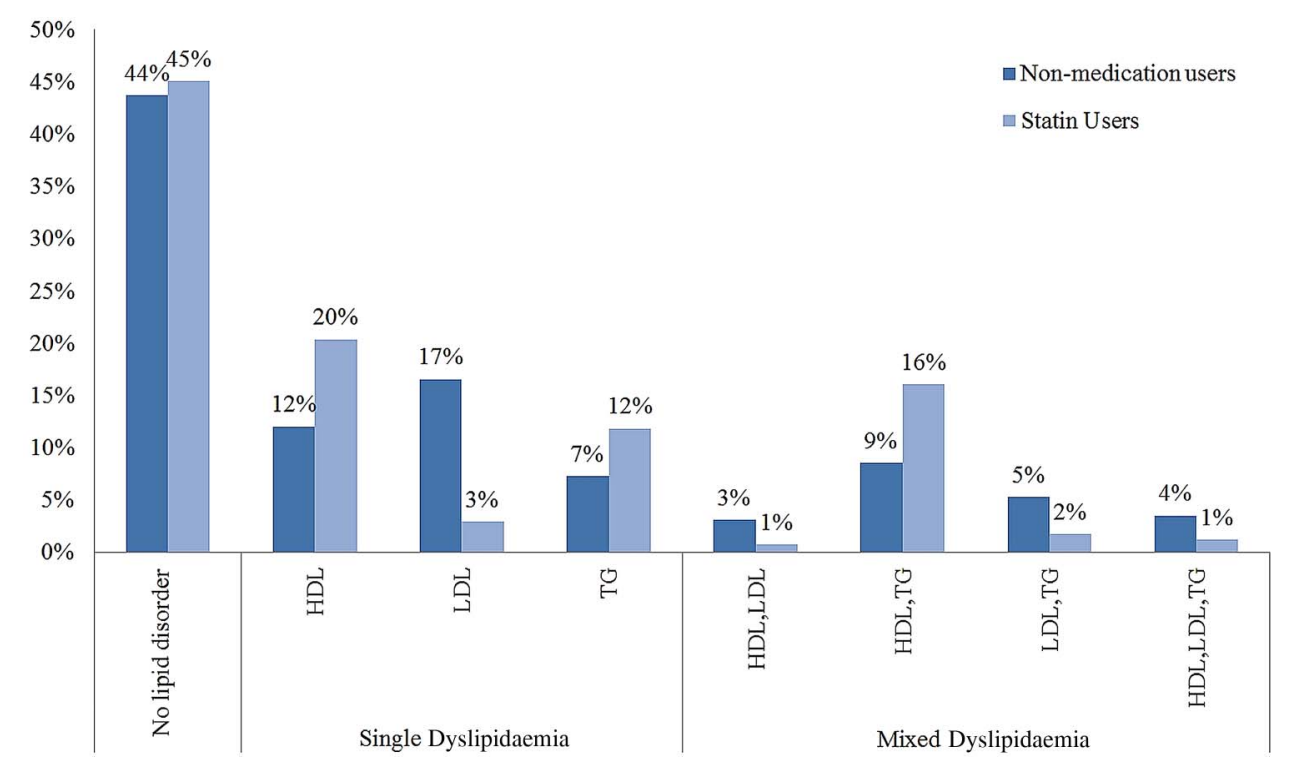

Figure 2 Lipid disorders in statin users and non-medication users in patients of EMR primary care settings in Canada $(n=111726)$. This figure shows the prevalence of each lipid abnormality among statin users (light blue), and non-medication users (dark blue). All comparisons are significant at $p<0.0001$ and were obtained from the $\chi^{2}$ tests. EMR, electronic medical records of family physicians. 


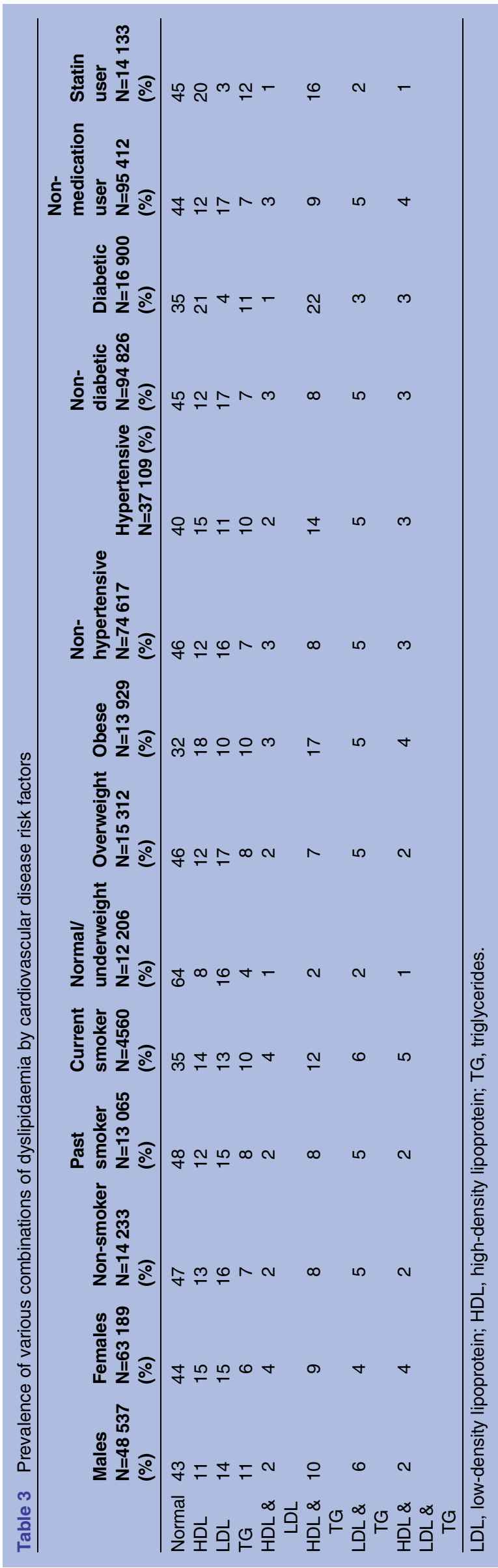

treatment of dyslipidaemia, where the main goal of treatment has been lowering the LDL levels using statins. ${ }^{2} 11$ The comparison of statin users with non-medication users in our study shows the fulfilment of this approach by lowering LDL-C levels. All lipid measurements encompassing LDL, including TC and ratio dyslipidaemia, are dramatically lower in statin users. On the other hand, other forms of dyslipidaemia (TG and HDL) are not only more common than LDL dyslipidaemia, but they also have a higher prevalence in these patients than in non-medication users. A similar trend is observed for mixed dyslipidaemia where all combinations of dyslipidaemia, that include LDL, are less prevalent in statin users; whereas the opposite effect is seen for those who do not encompass LDL (figure 2). As demonstrated in table 4, adjusting the findings for other variables does not seem to change this trend. These observations are overall consistent with previous reports that statins exert their effect through lowering LDL and their influence on other lipid components is minimal. Similar to our results are the findings by Colquhoun et $a \hat{l}^{35}$ on Australian patients who were treated primarily with statins and among whom nearly one-third had mixed dyslipidaemia after medication therapy. Although lipid modification therapy considerably improved LDL-C goal attainment, a large proportion of the patients in that study, similar to our study, did not achieve normal HDL-C and TG levels. ${ }^{35}$

The figures in our study, however, are lower than those from several other reports. Laforest $e t a l^{36}$ report that, of the total 2544 patients treated with statins in France, $51 \%$ and $32 \%$ of the participants had single and mixed dyslipidaemia, respectively; and, the figures were much higher for high-risk patients. In another study in France, $83 \%$ of the 946 patients treated for dyslipidaemia had single lipid disorders and $38 \%$ had mixed dyslipidaemia. ${ }^{37}$ The patients from those studies were selected directly from general practice clinics and their sample sizes were relatively lower, which may account for the higher prevalence of dyslipidaemia.

The 2013 ACC/AHA guidelines, ${ }^{38}$ which have the most recent available regulation of blood cholesterol to prevent CVDs, has similar recommendations to previous guidelines, except for adults aged 75 years and above. This group is recommended against the use of statins in the prevention of primary and secondary CVDs due to a lack of evidence on the effectiveness of such therapies in this target group. To further evaluate this recommendation, the same multinomial regression analysis was performed only for individuals aged over 75 years. Our findings were similar to the results from the analysis of the overall population; that is, statins are found to be effective on lowering LDL-C levels, however, they have minor effects on mixed dyslipidaemia (data not shown). Thus, it can be concluded that the lack of evidence for CVD protection in this group is either due to the shortage of clinical trials conducted on them, or because of the insufficient alternative beneficial effects of statins in 
Table 4 Single and mixed dyslipidaemia and some associated factors in patients of EMR primary care settings in Canada ( $\mathrm{n}=111726$ )

\begin{tabular}{|c|c|c|c|c|c|c|c|}
\hline & HDL & LDL & TG & HDL \& LDL & HDL \& TG & LDL \& TG & HDL \& LDL \& TG \\
\hline Gender (F) & 1.45 (1.39 to 1.51$)$ & $0.99(0.95 \text { to } 1.02)^{\mathrm{NS}}$ & 0.55 (0.53 to 0.58$)$ & 2.17 (2.00 to 2.36$)$ & $1.03(0.99 \text { to } 1.08)^{\mathrm{NS}}$ & 0.58 (0.55 to 0.62$)$ & $1.61(1.50$ to 1.74$)$ \\
\hline Age & 0.98 (0.98 to 0.99$)$ & 1.01 (1.01 to 1.01$)$ & $1.00(1.00 \text { to } 1.00)^{\mathrm{a}}$ & 0.98 (0.98 to 0.99$)$ & 0.99 (0.99 to 0.99$)$ & $1.00(1.00 \text { to } 1.00)^{\mathrm{NS}}$ & 0.99 (0.99 to 0.99$)$ \\
\hline $\begin{array}{l}\text { Previous } \\
\text { smoker }\end{array}$ & $0.98(0.91 \text { to } 1.06)^{\mathrm{NS}}$ & $0.98(0.91 \text { to } 1.05)^{\mathrm{NS}}$ & $0.99(0.90 \text { to } 1.09)^{\mathrm{NS}}$ & $1.14(0.96 \text { to } 1.36)^{\mathrm{NS}}$ & $1.00(0.91 \text { to } 1.11)^{\mathrm{NS}}$ & $0.96(0.86 \text { to } 1.08)^{\mathrm{NS}}$ & $1.10(0.92 \text { to } 1.31)^{\mathrm{NS}}$ \\
\hline $\begin{array}{l}\text { Current } \\
\text { smoker }^{*}\end{array}$ & $1.42(1.27$ to 1.58$)$ & $1.19(1.07 \text { to } 1.33)^{\mathrm{a}}$ & $1.59(1.40$ to 1.81$)$ & 2.61 (2.14 to 3.18 ) & $1.90(1.68$ to 2.15$)$ & 1.45 (1.23 to 1.69$)$ & 3.52 (2.93 to 4.22 ) \\
\hline Overweight $†$ & $1.93(1.77$ to 2.10$)$ & 1.55 (1.45 to 1.66$)$ & 2.06 (1.85 to 2.30$)$ & 3.15 (2.60 to 3.81$)$ & 3.68 (3.21 to 4.22$)$ & 2.89 (2.51 to 3.34$)$ & 3.79 (3.06 to 4.71$)$ \\
\hline Obese† & 3.63 (3.34 to 3.95 ) & $1.54(1.42$ to 1.67$)$ & 3.53 (3.16 to 3.94$)$ & 5.65 (4.68 to 6.83$)$ & $9.39(8.24$ to 10.71$)$ & 4.55 (3.93 to 5.26$)$ & $9.16(7.46$ to 11.26$)$ \\
\hline Rural residence & $1.12(1.07$ to 1.17$)$ & $0.95(0.91 \text { to } 1.00)^{\mathrm{a}}$ & $1.24(1.17$ to 1.30$)$ & $1.04(0.95 \text { to } 1.14)^{\mathrm{NS}}$ & $1.34(1.27$ to 1.41$)$ & $1.06(0.99 \text { to } 1.14)^{\mathrm{NS}}$ & $1.33(1.23$ to 1.44$)$ \\
\hline Diabetes & 2.12 (2.01 to 2.23$)$ & $0.35(0.32$ to 0.38$)$ & 1.51 (1.41 to 1.61$)$ & $0.72(0.62$ to 0.84$)$ & 3.05 (2.89 to 3.22$)$ & $0.70(0.63$ to 0.78$)$ & $1.11(0.99 \text { to } 1.24)^{\mathrm{NS}}$ \\
\hline Hypertension & $1.12(1.06$ to 1.18$)$ & $0.85(0.81$ to 0.90$)$ & $1.50(1.41$ to 1.60$)$ & $0.96(0.86 \text { to } 1.07)^{\mathrm{NS}}$ & 1.45 (1.37 to 1.54$)$ & $1.18(1.09-1.28)$ & $1.29(1.17$ to 1.42$)$ \\
\hline $\begin{array}{l}\text { Cardiovascular } \\
\text { disease }\end{array}$ & 1.25 (1.19 to 1.32$)$ & $0.89(0.85$ to 0.94$)$ & $1.08(1.02 \text { to } 1.15)^{\mathrm{a}}$ & $1.14(1.02 \text { to } 1.27)^{\mathrm{a}}$ & $1.26(1.19$ to 1.34$)$ & $0.98(0.91 \text { to } 1.07)^{\mathrm{NS}}$ & $1.08(0.98 \text { to } 1.19)^{\mathrm{NS}}$ \\
\hline $\begin{array}{l}\text { Drugs with } \\
\text { unintended lipid } \\
\text { effects }\end{array}$ & $1.09(1.03 \text { to } 1.14)^{\mathrm{a}}$ & $1.04(0.99 \text { to } 1.09)^{\mathrm{NS}}$ & $1.02(0.96 \text { to } 1.08)^{\mathrm{NS}}$ & $1.08(0.98 \text { to } 1.20)^{\mathrm{NS}}$ & $1.03(0.97 \text { to } 1.08)^{\mathrm{NS}}$ & $1.03(0.95 \text { to } 1.11)^{\mathrm{NS}}$ & 1.18 (1.08 to 1.29$)$ \\
\hline $\begin{array}{l}\text { Statin } \\
\text { monotherapy }\end{array}$ & 1.44 (1.36 to 1.53$)$ & $0.18(0.16$ to 0.20$)$ & 1.18 (1.10 to 1.27$)$ & $0.30(0.24$ to 0.36$)$ & 1.30 (1.22 to 1.38$)$ & 0.28 (0.24 to 0.32$)$ & 0.31 (0.26 to 0.37$)$ \\
\hline \multicolumn{8}{|c|}{$\begin{array}{l}\text { Figures are ORs ( } 95 \% \text { Cls). } \\
\text { All the presented ORs are significant at } p<0.0001 \text { except for: } \\
{ }^{2} p<0.05 \text {. } \\
\text { NS, not significant ( } p>0.05) \text {. } \\
\text { *Smoking status was compared with non-smokers. } \\
\text { tObesity status was compared with normal and underweight individuals. Population with no lipid disorders was considered as the base in the analysis. } \\
\text { EMR, electronic medical records of family physicians; HDL, high-density lipoprotein; LDL, low-density lipoprotein; TG, triglycerides. }\end{array}$} \\
\hline
\end{tabular}


the elderly. This conclusion may possibly be determined or revised following the conduct of future clinical trials.

There are several limitations in our study. We performed a cross-sectional study using EMRs of primary care settings in Canada to provide a snapshot of single and mixed dyslipidaemia in real practice. Our analyses showed $36 \%, 17 \%$, and $3 \%$ of the study population had abnormalities in one, two and three lipid components, respectively. It should be taken into consideration that the actual figure could be higher as the real prevalence of dyslipidaemia could be masked due to the effect of medication therapy. Furthermore, the patients in our study were selected from the Canadian population under primary care who had a lipid profile conducted by their family physician; generally speaking, a population with higher morbidity and, thus, the finding may not represent the general Canadian population. Moreover, this cross-sectional study using secondary data does not provide information to identify if the lipid test was requested according to the Canadian guidelines or other factors including patient request or a clinical suspicion by the family physician.

The study also does not provide information on patient adherence to lipid-lowering medication and the effectiveness of this medication over a long-term period; however, several studies, including systematic reviews, have shown the effectiveness of statin use during a shortterm period. ${ }^{39}$ Further, we did not have any data on some of the life-style factors that are associated with lipid levels, such as diet and physical activity. Finally, our data only corresponds to the population in EMR primary care settings of the Canadian Primary Care Sentinel Surveillance Network. Many systematic reviews on the effects and cost-effectiveness of computerised decisionsupport systems, including EMRs, have shown a significant benefit of these systems on practitioners' performance outcomes, whereas they have not found that the use of computerised decision-support systems consistently improve the process of care measures and patient outcomes. ${ }^{40} 41$

\section{CONCLUSION}

Our analysis indicates that a significant number of patients of EMRs primary care settings in Canada are suffering from single and mixed dyslipidaemia. The findings also demonstrate that the lipid management in a primary care setting is mainly focused on monotherapy of statins as recommended by Canadian guidelines. Although the statin therapy has significantly attained its LDL level goals among statin users, a significant number of these patients do not obtain the recommended levels for other components of lipids (12\% TG; $20 \%$ HDL dyslipidaemia), and the overall portion of mixed dyslipidaemia remains the same $(\sim 20 \%)$ among treated and untreated patients. This finding should be taken into consideration in clinical settings and possibly be used to revise the current guidelines for the treatment and prevention of CVDs, since statin-user patients can still remain unknowingly at high risk for future events from lipid components other than LDL-C. Further studies are required to determine the best therapeutic approaches that can be used for these patients.

Contributors SA and EA-E were involved in the study design, manuscript writing and statistical analysis; SA and TW were involved in the data collection; SA, EA-E, MG, PD, TW and MM were involved in the interpretation of the results; MG, PD, TW and MM were involved in the critical comments on the manuscript.

Funding This research did not receive any specific grant from any funding agency in the public, commercial or not-for-profit sectors.

\section{Competing interests None declared.}

Ethics approval Health Research Ethics Authority (HREA) of Newfoundland and Labrador.

Provenance and peer review Not commissioned; externally peer reviewed.

Data sharing statement No additional data are available.

Open Access This is an Open Access article distributed in accordance with the Creative Commons Attribution Non Commercial (CC BY-NC 4.0) license, which permits others to distribute, remix, adapt, build upon this work noncommercially, and license their derivative works on different terms, provided the original work is properly cited and the use is non-commercial. See: http:// creativecommons.org/licenses/by-nc/4.0/

\section{REFERENCES}

1. World Health Organization. The top 10 causes of death. May 2014 http://www.who.int/mediacentre/factsheets/fs310/en/ (accessed 10 Feb 2015).

2. Public health agency of Canada, Canadian institute for health information, Heart and stroke foundation of Canada, Statistics Canada. Tracking heart disease and stroke in Canada. 2009. http:// www.phac-aspc.gc.ca/publicat/2009/cvd-avc/pdf/cvd-avs-2009-eng. pdf (accessed 10 Feb 2015).

3. Smith SC Jr, Allen J, Blair SN, et al. AHA/ACC guidelines for secondary prevention for patients with coronary and other atherosclerotic vascular disease: 2006 update: endorsed by the National Heart, Lung, and Blood Institute. Circulation 2006;113:2363-72.

4. Yusuf S, Hawken S, Ounpuu S, et al. INTERHEART Study Investigators. Effect of potentially modifiable risk factors associated with myocardial infarction in 52 countries (the INTERHEART study): case-control study. Lancet 2004;364:937-52.

5. Musunuru K. Atherogenic dyslipidemia: cardiovascular risk and dietary intervention. Lipids 2010;45:907-14.

6. Wilson PW. High-density lipoprotein, low-density lipoprotein and coronary artery disease. AmJ CardioL 1990;66:A7-10.

7. Gordon T, Castelli WP, Hjortland MC, et al. High density lipoprotein as a protective factor against coronary heart disease. The Framingham Study. Am J Med 1977;62:707-14.

8. Gordon DJ, Probstfield JL, Garrison RJ, et al. High-density lipoprotein cholesterol and cardiovascular disease. Four prospective American studies. Circulation 1989;79:8-15.

9. Sarwar N, Danesh J, Eiriksdottir G, et al. Triglycerides and the risk of coronary heart disease: 10,158 Incident cases among 262,525 participants in 29 Western prospective studies. Circulation 2007;115:450-8.

10. Morrison A, Hokanson JE. The independent relationship between triglycerides and coronary heart disease. Vasc Health Risk Manag 2009;5:89-95

11. Genest J, McPherson R, Frohlich J, et al. Canadian cardiovascula society/Canadian guidelines for the diagnosis and treatment of dyslipidemia and prevention of cardiovascular disease in the adult2009 recommendations. Can J Cardiol 2009;25:567-79.

12. Fazio S. Management of mixed dyslipidemia in patients with or at risk for cardiovascular disease: a role for combination fibrate therapy. Clin Ther 2008;30:294-306.

13. Baigent C, Blackwell L, Emberson J, et al, Cholesterol Treatment Trialists' Collaboration. Efficacy and safety of more intensive lowering of LDL cholesterol: a meta-analysis of data from 170,000 participants in 26 randomised trials. Lancet 2010;376:1670-81. 
14. Miller M, Cannon CP, Murphy SA, et al. Impact of triglyceride levels beyond low-density lipoprotein cholesterol after acute coronary syndrome in the PROVE IT-TIMI 22 trial. J Am Coll Cardiol 2008;51:724-30.

15. Barter P, Gotto AM, LaRosa JC, et al. HDL cholesterol, very low levels of LDL cholesterol, and cardiovascular events. $N$ Engl J Med 2007;357:1301-10.

16. Wolfram RM, Brewer HB, Xue Z, et al. Impact of low high-density lipoproteins on in-hospital events and one-year clinical ouomes in patients with non-ST-elevation myocardial infarction acute coronary syndrome treated with drug-eluting stent implantation. Am J Cardiol 2006;98:711-7.

17. Turner RC, Millns $\mathrm{H}$, Neil HA, et al. Risk factors for coronary artery disease in non-insulin dependent diabetes mellitus: United Kingdom Prospective Diabetes Study (UKPDS: 23). BMJ 1998;316:823-8.

18. Chapman MJ, Ginsberg HN, Amarenco P, et al. Triglyceride-rich lipoproteins and high-density lipoprotein cholesterol in patients at high risk of cardiovascular disease: Evidence and guidance for management. Eur Heart $J$ 2011;32:1345-61.

19. Joffers M, Shields M, Tremblay MS, et al. Dyslipidemia prevalence, treatment, control, and awareness in the Canadian Health Measures Survey. Can J Public Health 2013;104:e252-7.

20. Petrella RJ, Merikle E, Jones J. Prevalence and treatment of dyslipidemia in Canadian primary care: a retrospective cohort analysis. Clin Ther 2007;29:742-50.

21. De Backer G, Ambrosioni E, Borch-Johnsen K, et al. European guidelines on cardiovascular disease prevention in clinical practice: Third joint task force of European and other societies on cardiovascular disease prevention in clinical practice (constituted by representatives of eight societies and by invited experts). Eur $J$ Cardiovasc Prev Rehabil 2003;10:S1-S10.

22. Chait A, Albers JJ, Brunzell JD. Very low density lipoprotein overproduction in genetic forms of hypertriglyceridaemia. Eur J Clin Invest 1980;10:17-22.

23. Birtwhistle R, Keshavjee K, Lambert-Lanning A, et al. Building a pan-Canadian primary care sentinel surveillance network: initial development and moving forward. J Am Board Fam Med 2009;22:412-22.

24. Asghari S, Aref-Eshghi E, Hurley O, et al. Does the prevalence of dyslipidemias differ between Newfoundland and the rest of Canada? Findings from the Electronic Medical Records of the Canadian Primary Care Sentinel Surveillance Network. Front Cardiovasc Med 2015;2:1.

25. Aref-Eshghi E, Leung J, Godwin M, et al. Low density lipoprotein cholesterol control status among Canadians at risk for cardiovascula disease: findings from the Canadian Primary Care Sentinel Surveillance Network Database. Lipids Health Dis 2015;14:60.

26. Devine $\mathrm{S}$, West $\mathrm{S}$, Andrews $\mathrm{E}$, et al. The identification of pregnancies within the general practice research database. Pharmacoepidemiol Drug Saf 2010;19:45-50.

27. Statistics Canada. Population and dwelling counts for Canada, census metropolitan areas, census agglomerations and census subdivisions (municipalities), 2011 and 2006 censuses. January 2014. http://www12.statcan.gc.ca/census-recensement/2011/dp-pd/
hlt-fst/pd-pl/Table-Tableau.cfm?LANG=Eng\&T=203\&CMA=001\& $\mathrm{S}=0 \& \mathrm{O}=\mathrm{D} \& \mathrm{RPP}=25$ (accessed $10 \mathrm{Feb} 2015$ )

28. Lindroth M, Robert L, Mikael L, et al. Cardiovascular risk factors differ between rural and urban Sweden: the 2009 Northern Sweden MONICA cohort. BMC Public Health 2014;14:825.

29. Kadhim-Saleh A, Green M, Williamson T, et al. Validation of the diagnostic algorithms for 5 chronic conditions in the Canadian Primary Care Sentinel Surveillance Network (CPCSSN): a Kingston Practice-based Research Network (PBRN) report. J Am Board Fam Med 2013;26:159-67.

30. Herrett E, Thomas SL, Schoonen WM, et al. Validation and validity of diagnoses in the General Practice Research Database: a systematic review. Br J Clin Pharmacol 2010;69:4-14.

31. Gulliford MC, Charlton J, Ashworth M, et al. eCRT Research Team. Selection of medical diagnostic codes for analysis of electronic patient records. Application to stroke in a primary care database. PLOS ONE 2009;4:e7168.

32. Jones PH, Nair R, Thakker KM. Prevalence of dyslipidemia and lipid goal attainment in statin-treated subjects from 3 data sources: a retrospective analysis. J Am Heat Assoc 2012;1:e001800.

33. Mantel-Teeuwisse AK, Kloosterman JM, Maitland-van der Zee AH, et al. Drug-induced lipid changes: a review of the unintended effects of some commonly used drugs on serum lipid levels. Drug Safety 2001;24:433-56.

34. Rubins HB, Robins SJ, Collins D, et al. Distribution of lipids in 8500 men with coronary artery disease. Department of Veterans Affairs HDL Intervention Trial Study Group. Am J Cardiol 1995;75:1196-1201.

35. Colquhoun D, Chirovsky D, Sazonov V, et al. Prevalence of mixed dyslipidemia among Australian patients undergoing lipid-modifying therapy. Exp Clin Cardiol 2013;18:e32-6.

36. Laforest L, Ambegaonkar BM, Souchet T, et al. Mixed dyslipidemia in primary care patients in France. Vasc Health Risk Manag 2012;8:247.

37. Van Ganse E, Laforest L, Burke T, et al. Mixed dyslipidemia among patients using lipid-lowering therapy in French general practice: an observational study. Clin Ther 2007;29:1671-81.

38. Stone NJ, Robinson JG, Lichtenstein AH, et al. ACC/AHA guideline on the treatment of blood cholesterol to reduce atherosclerotic cardiovascular risk in adults: a report of the American College of Cardiology/American Heart Association Task Force on Practice Guidelines. J Am Coll Cardiol 2014;63(25 Pt B):2889-934.

39. Rosenson RS, Underberg JA. Systematic review: evaluating the effect of lipid-lowering therapy on lipoprotein and lipid values. Cardiovasc Drugs Ther 2013;27:465-79.

40. Main C, Moxham T, Wyatt JC, et al. Computerized decision support systems in order communication for diagnostic, screening or monitoring test ordering: systematic reviews of the effects and cost-effectiveness of systems. Health Technol Assess 2010;14:1-227.

41. Hemens BJ, Holbrook A, Tonkin M, et al. Computerized clinical decision support systems for drug prescribing and management: a decision-maker-researcher partnership systematic review. Implement Sci 2011;6:89. 